\title{
La barrière placentaire : structure, résistance, asymétrie
}

\author{
J.C. Challier \\ Université Pierre-et-Marie Curie, biologie de la reproduction, bât. A, 7, quai Saint-Bernard, \\ 75252 Paris Cedex 05, France
}

(14 réunion du groupe Développement INRA, Clermont-Ferrand, 25-27 mai 1988)

\begin{abstract}
Résumé - La notion de barrière placentaire a été réexaminée en tenant compte des données récentes d'ultrastructure et des nouvelles observations de la physiologie des échanges. D'un point de vue ultrastructural, l'effet barrière repose sur la continuité du syncytiotrophoblaste, la porosité des lames basales ainsi que sur la charge électrique des membranes plasmiques et basales. La continuité de la phase aqueuse pour les passages diffusionnels s'effectue par les espaces intercellulaires, les fenestrations (rat, lapin) et des canaux transcellulaires (cobaye). Mais les structures qui assurent cette continuité dans le syncytiotrophoblaste humain ne sont pas apparentes. La diffusion est basée sur les propriétés physico-chimiques des molécules (taille, charge électrique, liposolubilité). Pour la taille des molécules, les placentas hémochoriaux (avec une population de pores de rayon $10 \mathrm{~nm}$ ) apparaissent bien moins sélectifs que les placentas épithéliochoriaux. La capactié métabolique des différents types cellulaires du placenta (trophoblaste, macrophages) participe à cet effet barrière en catabolisant ou en transformant certains substrats. De même, l'asymétrie du trophoblaste dans la distribution des enzymes, des transporteurs et des récepteurs entre les membranes plasmiques apicale (face maternelle) et basale (face fœtale) et dans les phénomènes sécrétoires contribue au maintien de compartiments fœtal et maternel distincts. La polarité fonctionnelle du trophoblaste se manifeste dans le métabolisme (corticostéroïdes), le transport de certains nutriments (acides aminés), de certains ions (fer) et de la plupart des sécrétions endocrines (hCG, hPL, SP1).
\end{abstract}

barrière - placenta - transport

Summary - The placental barrier : structure, resistance, asymmetry. The concept of placental barrier has been evaluated using recent advances in ultrastructure and in transport physiology. On a structural basis, the barrier effect is grounded by the syncytiotrophoblast continuity, and by basal and plasma membrane's electrical charges and by basement membrane porosity. The aqueous phase continuity for diffusion operates through intercellular gap, fenestrations (rat, rabbit) and transcellular channels (guinea pig). However, these connections are not apparent in the human syncytiotrophobast. For the molecular size selectivity, the hemochorial placentas with a pore radius of $10 \mathrm{~nm}$ appear much less selective than the epitheliochorial ones. The metabolic capacity of the placental cells (trophoblast, macrophages) participates to the barrier effect by metabolizing or by converting some substrates. Similarly, trophoblast asymmetry in the location of enzymes, carriers and receptors on outer (maternal side) and on basal (fetal side) plasma membranes, and in the release of secreto$r y$ products, contributes to maintain separate fetal and maternal compartments. The functional polarity of trophoblast is expressed in metabolism (corticostéroids), nutrients (amino acids) and ions (iron) transport, and most of its secretions ( $h P L, h C G, S P 1)$.

barrier - placenta - transport 


\section{INTRODUCTION}

La classification des couches de tissus qui séparent le sang maternel du sang fotal a été établie par Grosser (1909, 1927). Elle est à l'origine de la notion de barrière placentaire. Flexner \& Gellhorn (1942) ont démontré ensuite l'existence d'un effet barrière sur le passage du sodium en relation avec le nombre de couches tissulaires dans les placentas épithéliochorial, endothéliochorial et hémochorial. Cette relation n'a pas pu être étendue au nombre de couches de trophoblaste dans les placentas hémomonochorial, hémodichorial et hémotrichorial (Faber, 1973). De nouvelles causes structurales et de nouveaux effets barrière ont été mis en évidence ces dernières années et la notion même de barrière biologique s'est élargie. Le rôle des structures de la membrane placentaire dans les relations fœeto-maternelles doit donc être reconsidéré.

L'effet barrière a un sens restrictif qui s'applique particulièrement bien à l'embryon et au fœtus, car ces derniers sont dépourvus de systèmes immunitaire et de détoxification. Cet effet a des bases structurales, mais il met aussi en œuvre des processus cataboliques. A l'inverse, l'effet barrière appliqué à un organe qui approvisionne le fœtus en nutriments et qui excrète les produits de son métabolisme peut paraître paradoxal. En fait, les membranes biologiques ont mis en place des systèmes spécifiques de transport et des récepteurs capables d'accroître le passage des molécules, et au besoin de les réguler. La distribution asymétrique des transporteurs et celle des récepteurs sont associées à l'effet barrière. II en va de même de la polarisation des sécrétions du trophoblaste, lesquelles apparaissent préférentiellement dans la circulation maternelle.
Nous développerons cette conception de la barrière placentaire en examinant successivement : les indices structuraux de la résistance membranaire, leur évolution au cours du développement, les manifestations expérimentales de l'effet barrière. Les facteurs limitant le passage des molécules, qui ne sont pas directement liés à la structure ou à l'activité de la membrane placentaire, ne seront pas pris en considération.

\section{INDICES STRUCTURAUX DE LA RÉSIS- TANCE MEMBRANAIRE}

\section{Présence de g/ycocalyx}

La surface du syncytium trophoblastique humain est hérissée de microvillosités. Elle est recouverte d'un revêtement glycoprotéique appelé glycocalyx, riche en charges anioniques qui fixent le fer colloïdal ( $\mathrm{Nel}$ son et al., 1976), le rouge de ruthénium (Sideri et al., 1982) et la ferritine cationique (Kawagoe et al., 1981). Ces charges sont disposées en amas discontinus sur la surface syncytiale (Kawagoe et al., 1981) et forment une barrière électrostatique. Dixsept sialoglycoprotéines ont été identifiées (Wada et al., 1977) qui proviennent de la sialylation des oligosaccharides des glycoprotéines de la membrane plasmique. La sialylation des glycopeptides contenant de la glucosamine est plus importante dans le syncytiotrophoblaste que dans les cellules du fœtus (Whyte \& Locke, 1978). Martin et al. (1974) ont observé sur le syncytium la présence d'acide hyaluronique, laquelle n'a pas été confirmée. La liaison avec des lectines montre l'existence d'autres constituants des glycosaminoglycanes : manno- 
et gluco-pyranosides, et $\mathrm{N}$-acétylglucosamine. Elle indique peu de fucose et révèle paradoxalement peu d'acide $\mathrm{N}$-acétylneuraminique (Whyte, 1980). II est probable que I'hCG, la phosphatase alcaline et la 5nucléotidase qui sont des glycoprotéines membranaires liant la concavaline $A$ font partie du glycocalyx. Ce dernier est donc constitué essentiellement de sialoglycoprotéines, de glycosaminoglycanes et certainement de glycolipides. Un dépôt de fibrine s'adjoint au glycocalyx dans les zones subchoriales de l'espace intervilleux en fin de gestation. Son effet r'est pas défini.

La surface de l'endothélium des capillaires fœtaux chez le cobaye et dans l'espèce humaine présente également des groupements anioniques et elle ne diffère pas des autres endothéliums puisqu'elle fixe la peroxydase cationique (Sideri et al., 1987; Sibley et al., 1981; Kaufmann et al., 1982) et l'hydroxyde de lanthane (Kaufmann et al., 1987). La peroxydase cationique est identifiée sur toute la longueur des jonctions interendothéliales dans le placenta humain (Sideri et al., 1987), de même que chez le cobaye (Sibley et al., 1981).

\section{Continuité du syncytiotrophoblaste}

Chez les primates, aucune discontinuité structurale de nature à assurer un passage transtrophoblastique n'a été clairement démontrée. Les canaux observés par Gammal (1985) chez le macaque sont une exception. Cependant, ces canaux ne sont pas en continuité avec la membrane plasmique basale du syncytium et ils sont observés surtout dans les cellules qui font saillie dans l'espace intervilleux, ce qui jette un doute sur leur rôle dans le passage transplacentaire. Les vacuoles et les canaux transcellulaires induits lors d'un mouvement osmotique d'eau dans le placenta hémomonochorial de cobaye (Kaufmann et al., 1982) constituent peut-être un réseau transtrophoblastique de communications. Pour Kaufmann et al. (1987), il correspondrait dans les conditions isoosmotiques à un réseau tubulaire de 15 $\mathrm{nm}$ de diamètre dans lequel pénètre le lanthanum. Dans le placenta hémodichorial du lapin, Thornburg \& Faber (1976) ont montré des fenestrations du syncytium qui rappellent celles observées dans l'endothélium des glomérules du rein.

Des jonctions cellulaires de type occludens (macula, fascia) ont été mises en évidence sur la face fotale du syncytium et des desmosomes incomplets dans le cytoplasme (De Virgilis et al., 1982; Metz et al., 1979; Reale et al., 1980) en plus des jonctions communicantes. Les desmosomes proviennent de la fusion du cytotrophoblaste avec le syncytium (Reale et al., 1980); les maculae occludentes seraient situées au niveau des points de contact qui se forment entre les villosités (Metz et al., 1979).

Bien qu'un certain nombre de structures tubulaires ou vésiculaires aient été observées dans le trophoblaste humain, il ne semble pas qu'elles forment un réseau transtrophoblastique continu. De plus, il n'y a pas, dans les conditions iso-osmotiques, de compartiment paracellulaire (canaux transtrophoblastiques) susceptible de jouer un rôle dans les échanges transmembranaires. Les vésicules de pinocytose (micro- et macropinocytose) pourraient former un système transcellulaire discontinu. Elles sont nombreuses mais elles restent localisées près des microvillosités du syncytium. II est donc peu probable qu'elles permettent aux macromolécules de traverser cette assise cellulaire. Les nombreuses vésicules d'endocytose à couche de clathrine (coated vesicles) situées près des microvillosités pourraient intervenir 
également, mais de façon très sélective et sans nécessairement aboutir à un passage transcellulaire. II faut en référer aux études avec traceurs qui ne sont qu'évoquées ici.

\section{Structure et charge électrique des membranes basales}

La membrane basale du syncytium est composée de 3 couches : la lamina rara, la lamina densa et la couche réticulée. La lamina rara contient une sialoglycoprotéine, la fibronectine, et de la laminine qui relient les cellules épithéliales au collagène de la membrane basale. Des protéoglycanes porteuses de groupements sulfates sont présentes en bordure de la

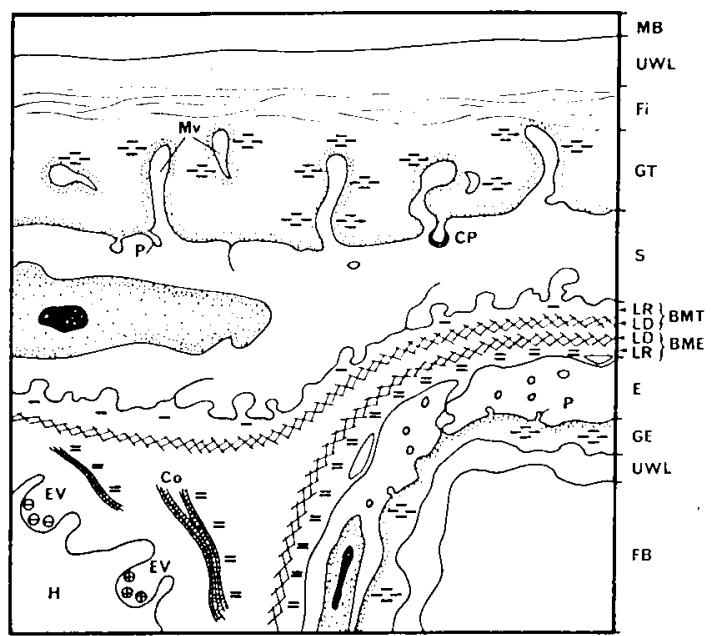

Fig. 1. Barrières dans le placenta humain à terme. BMT : membrane basale du trophoblaste; BME : membrane basale de l'endothélium; Co : fibre de collagène; $\mathrm{CP}$ : vésicule à clathrine (coated pit); $E$ : endothélium; Ev : vacuole d'endocytose; Fi : fibrine; FB : sang fœtal; GE : glycocalyx endothélial; GT : glycocalyx trophoblastique; $H$ : histiocytes; LR : lamina rara; LD : lamina densa; $\mathrm{MB}$ : sang maternel; Mv : microvillosité; $P$ : vacuole de pinocytose; UWL : couche d'eau associée; $S$ : syncytiotrophoblaste. lamina densa. Elles contiennent des charges anioniques que l'on détecte dès le premier trimestre de gestation (Fig. 1). Elles sont attribuées à l'héparan-sulfate (King, 1985). A terme, elles ont disparu de la membrane basale du trophoblaste; quelques-unes sont observées dans la membrane basale de l'endothélium et associées au collagène du tissu interstitiel. Elles sont dues à des acides chondroïtinesulfate.

\section{Absorption par les histiocytes}

Les histocytes (ou cellules de Hofbauer) absorbent les substances qui arrivent jusqu'au conjonctif. Au cours de la perfusion des vaisseaux fœtaux du placenta de cobaye, la peroxydase traverse l'endothélium des capillaires, puis elle est phagocytée par les histiocytes (Kaufmann et al., 1982). De même, lors de courtes perfusions, la peroxydase cationique injectée dans la circulation fœtale ou maternelle du placenta humain, est retrouvée dans les histiocytes (Sideri et al., 1987). La peroxydase anionique administrée dans la circulation fœtale se fixe également dans les macrophages. En immobilisant les molécules qui atteignent le conjonctif, les histiocytes participent donc à l'effet barrière du trophoblaste. II ne semble pas qu'ils soient capables de discriminer les macromolécules selon leur charge électrique (Stenseth et al., 1983).

\section{Continuité de l'endothélium}

L'endothélium des capillaires constitue une couche continue chez les mammifères, excepté chez le rat où l'on observe des fenestrations nombreuses. Les jonctions intercellulaires chez le cobaye rap- 
pellent par leur structure les jonctions serrées (zonula occludens ou adherens).

Dans l'endothélium des capillaires humains, on observe des jonctions serrées de structure voisine (Heinrich et al., 1976; Sideri et al., 1983). Ces jonctions de l'endothélium, bien que de type serré, ne présentent pas de fusion continue du feuillet membranaire externe en microscopie électronique. En cryofracture, elles forment des lignes discontinues de particules. Ces jonctions sont généralement perméables aux substances de taille inférieure ou égale à $4 \mathrm{~nm}$. Un passage de lanthanum, de rayon égal à $0,4 \mathrm{~nm}$, ou de peroxydase, de rayon égal à $3 \mathrm{~nm}$, a été décelé à travers les capillaires fœto-placentaires chez le rat (Aoki et al., 1978), chez le cobaye (Firth et al., 1983; Kaufmann et al., 1987), dans l'espèce humaine (Sideri et al., 1987). Cependant, la ferritine de taille beaucoup plus importante $(11 \mathrm{~nm})$ n'apparaît pas dans les espaces subendothéliaux chez le cobaye (Sibley et al., 1983) et le lapin (Thornburg \& Faber, 1976).

Des vésicules de pinocytose marquées par la peroxydase sont observées dans l'endothélium des capillaires dans l'espèce humaine et chez le cobaye. Leur grand nombre indique qu'elles ont un rôle important dans le passage transendothélial.

\section{EVOLUTION DES BARRIÈRES AU COURS DU DÉVELOPPEMENT}

\section{Amincissement du syncytiotropho- blaste}

Le syncytiotrophoblaste humain a une épaisseur moyenne de $6,4 \mu \mathrm{m}$ à $26 \mathrm{se}$ - maines et $3,7 \mu \mathrm{m}$ à 42 semaines de grossesse. On note également une réduction de la taille des microvillosités vers 5 mois.

\section{Réduction numérique du cytotropho- blaste}

Dans l'espèce humaine, la membrane d'échange placentaire s'amincit au cours de la gestation. Cet amincissement provient en partie de l'incorporation des cellules trophoblastiques dans le syncytium. Le cytotrophoblaste occupe environ $5 \%$ de la surface totale placentaire à 2 mois de grossesse. II couvre moins de $1 \%$ de cette surface à terme (Snoeck, 1958). Dans le placenta de rat à 14 jours de gestation, on rencontre des cellules indifférenciées entre le syncytium I et la troisième couche trophoblastique; elles seront incorporées entre 14 et 16 jours de gestation dans l'assise de syncytium II (Metz, 1980). La même observation a été faite chez le cobaye par Kaufmann \& Davidoff (1977). Leur disparition réduit l'épaisseur du trophoblaste.

\section{Formation de membranes vasculo- syncytiales}

Les membranes vasculo-syncytiales apparaissent vers la $32^{e}$ semaine de grossesse (Fox, 1967). Ce sont des zones où le syncytium est mince, dépourvu de noyau et contigu à l'endothélium capillaire. Les noyaux se regroupent entre ces zones et forment parfois des amas nucléaires. Cette tendance qu'ont les vaisseaux à se rapprocher et à s'introduire dans le trophoblaste au cours du développement est observée aussi chez la brebis. 


\section{Modification des membranes basales}

La membrane basale du trophoblaste est formée très précocement dans l'espèce humaine. Elle s'épaissit vers la $34 \mathrm{e}$ semaine de grossesse (Fox, 1978). Celle de l'endothélium est encore absente ou incomplète entre 7 et 12 semaines (King, 1985).

En début de gestation, l'arrangement régulier des protéoglycanes constitue à la fois un réseau de filtration et une barrière électrostatique. Les fibres de collagène de type IV disposées en réseau dans la lamina densa (Farquhar et al., 1982) pourraient effectuer une filtration des produits en transit. En fin de gestation, la diminution des charges anioniques dans la membrane basale du syncytium favorise certainement le passage des macromolécules de même charge, mais la structure de filtration subsiste. Le collagène et la membrane basale des capillaires conservent leur effet électrostatique.

\section{PREUVES EXPÉRIMENTALES D'UN EFFET BARRIÈRE}

\section{Résistance au passage des molécules hydrosolubles}

\section{Petites molécules polaires}

La résistance de la barrière placentaire au passage des molécules hydrosolubles dépend de leur poids moléculaire. L'eau tritiée et l'antipyrine, une molécule liposoluble, ont des transferts materno-fœtaux identiques in vivo chez le mouton (Meschia et al., 1967), chez le singe (Battaglia et al., 1968), et in vitro dans le placenta humain perfusé (Challier et al., 1985). Des molécules de liposolubilité si différente ne devraient pas avoir des transferts transplacentaires aussi proches. Un transport maximal des 2 molécules, ou moins vraisemblablement une limitation commune, peut expliquer ce résultat. En conséquence, il faut concevoir que le placenta oppose une résistance équivalente à leur passage, lequel est limité uniquement par le débit circulatoire. Des conclusions analogues ont été tirées d'expérimentations réalisées avec d'autres molécules de liposolubilité différente chez le lapin (Faber \& Hart, 1966) et chez le cobaye (Moll \& Kastendieck, 1977).

\section{Molécules de taille moyenne et macro- molécules}

\section{Pores physiologiques}

La résistance de la membrane placentaire s'accroît à mesure que la taille des molécules augmente. Dans le placenta épithéliochorial de brebis, une limitation du passage des molécules polaires sans charge électrique a été démontrée (Boyd et al., 1976). La taille des pores responsables de cette limitation est évaluée à $0,5 \mathrm{~nm}$, ce qui correspond à la taille des pores de la membrane plasmique des hématies : 0,35 $\mathrm{nm}$ (Solomon, 1968). Dans les placentas de lapin (Faber et al., 1971) et de cobaye (Hedley \& Bradbury, 1980), la taille des pores s'élève à $10 \mathrm{~nm}$ ou plus. II en est de même dans le placenta humain en perfusion (Challier et al., 1985). L'existence de 2 populations de pores : l'une à $0,3 \mathrm{~nm}$, l'autre à $10 \mathrm{~nm}$ dans le placenta de lapin (Stulc et al., 1969) n'a pas été confirmée. Les pores des placentas hémochoriaux ont donc une taille intermédiaire entre les 2 tailles de pores identifiés dans les ca- 
pillaires : $5-8 \mathrm{~nm}$ et $20-25 \mathrm{~nm}$ (Taylor \& Granger, 1983).

Le passage d'eau d'origine osmotique dans le placenta de brebis s'effectue par des pores de taille 3 fois plus élevée que les pores diffusionnels : $1,6 \mathrm{~nm}$ selon l'estimation de Stulc (1985). Cette observation montre que les passages d'eau et de moiecules polaires suivent apparemment des voies différentes.

\section{Charge électrique}

Les charges électriques du glycocalyx syncytial ou endothélial, des membranes basales, la différence de charge entre les milieux extracellulaire et intracellulaire ainsi que le potentiel transplacentaire peuvent intervenir selon leur importance et leur signe dans le passage placentaire.

Le transfert des électrolytes comme le sodium (rayon hydraté : $0,34 \mathrm{~nm}$ ) ou le chlore (rayon hydraté : $0,22 \mathrm{~nm}$ ) est fortement limité par rapport à l'urée chez la brebis (Conrad \& Faber, 1977), bien que les rayons de ces 3 substances soient très proches. La cause de cette limitation n'est pas la taille des ions, qui est inférieure à celle des pores, ni leur charge, puisque la charge opposée des 2 ions affecte peu leur transfert. Chez le lapin et le cobaye au contraire, les passages du sodium et du chlore sont analogues à celui de l'urée. De plus, comme ils s'insèrent parfaitement dans la relation transfert-coefficient de diffusion établie pour les substances non chargées électriquement (Faber et al., 1971), on doit admettre que leur transfert est subordonné à leur taille et non à leur charge. Des résultats similaires ont été obtenus dans le placenta humain perfusé (Dancis et al., 1981). L'étude du système d'échange proton-sodium, de celui des anions et $\mathrm{du}$ cotransport sodiumpotassium-chlorure mis en évidence sur les microvésicules syncytiales humaines devrait permettre de mieux comprendre le mécanisme de transport des ions (Schennan et Boyd, 1987).

Les macromolécules ont pour la plupart un point iso-électrique qui les rend anioniques au $\mathrm{pH}$ physiologique. Elles sont de ce fait soumises à l'effet des barrières électrostatiques. Nous avons montré que la peroxydase cationique traverse in vitro la barrière placentaire humaine dans les 2 directions : fœtus-mère et mère-fœtus (Sideri et al., 1987). Dans l'endothélium, elle emprunte les espaces interendothéliaux et les vésicules d'endocytose, et dans le syncytium, des vésicules ou des tubules. La peroxydase anionique est observée dans les tissus de la barrière trophoblastique uniquement lorsqu'elle est injectée dans la circulation fœtale. Un passage préférentiel de la peroxydase cationique par rapport à la peroxydase anionique a été mis en évidence chez le cobaye (Behre et al., 1987) qui souligne le rôle des barrières électrostatiques dans le placenta. On peut également concevoir que les traversées mèrefœtus et fœtus-mère s'opèrent par des voies différentes.

La différence de potentiel entre le fœetus et la mère peut affecter le passage des ions. Ces potentiels s'échelonnent de +15 $\mathrm{mV}$ (fœtus positif) chez le rat, à $0 \mathrm{mV}$ dans l'espèce humaine et chez le lapin, à -20 $\mathrm{mV}$ chez le cobaye et à $-50 \mathrm{mV}$ chez la brebis. Ces valeurs ne représentent pas nécessairement la différence de potentiel transplacentaire. En effet, lorsque des ions non présents normalement dans le sang sont administrés à la brebis, leurs concentrations fœtale et maternelle s'équilibrent presque et leur potentiel d'équilibre est seulement de quelques millivolts (Thornburg et al., 1979). Seules des mesures au niveau placentaire permettraient de répondre à cette question. Nous venons d'aborder cette question in vitro dans le 
placenta humain (Bara et al., 1988). II est clair qu'il existe un potentiel électrique faible $(-30 \mathrm{mV})$ au niveau de la membrane plasmique apicale du trophoblaste qui comprend un potentiel diffusionnel et un potentiel actif. Leur participation aux transports ioniques devra être précisée.

\section{Résistance au passage des molécules liposolubles}

Peu d'études ont été consacrées au passage des molécules liposolubles. Pour entrer dans les cellules, elles doivent se dissoudre dans la bicouche lipidique des membranes. Leur perméation diminue avec leur liposolubilité. Cette relation a été démontrée chez le cobaye et chez la brebis (Bissonnette et al., 1979) en pratiquant des injections en bolus. Dans les 2 espèces, le transfert normalisé (en éliminant le poids moléculaire des substances) met en évidence une perméabilité élevée du méthanol $(1,3)$, faible de l'eau (1) et de l'antipyrine $(0,5-0,6)$ et plus faible encore de l'acétamide $(0,29-0,11)$. La basse perméabilité de l'antipyrine semble remettre en cause le transport maximal des substances évoqué précédemment. II s'agit d'observations qui reflètent le transfert unidirectionnel (sang-placenta) et non le passage (sang maternel-placenta-sang fœetal) en état stable. Pour des raisons indéterminées, elles ne sont pas représentatives de la situation in vivo. Néanmoins, Wilkening et al. (1982) ont obtenu une clairance légèrement plus importante de l'éthanol par rapport à l'antipyrine en état stable. Existe$t-i l$ des "pores lipidiques" en plus des pores aqueux ? Cela reste à démontrer.

La résistance au passage des substances liposolubles peut avoir 3 causes annexes. Le franchissement de la couche externe d'eau associée à la membrane plasmique qui serait facilité par une liaison à l'albumine dans le cas des acides gras (Dancis et al., 1974) ou aux lipoprotéines dans le cas du cholestérol (Winkel et al., 1980). L'immobilisation des substances par une dissolution dans les lipides intracellulaires : les benzodiazépines, par exemple, sont accumulées en quantité très importante par le placenta sans qu'un métabolisme quelconque de ces substances ait pu être détecté (Guerre-Millo et al., 1982). Enfin dans le syncytiotrophoblaste, l'absence de continuité entre la bordure microvillositaire et la membrane plasmique basale du syncytium restreint certainement le passage des substances liposolubles de la face maternelle à la face fœtale par diffusion dans les lipides membranaires.

\section{BARRIÈRE MÉTABOLIQUE}

\section{Polarité de sécrétion}

Les sécrétions placentaires sont déversées en majeure partie dans la circulation maternelie. C'est le cas de l'hCG, d'hPL, de la SP1, de la PAPP-A (Bersinger et al., 1986), de la phosphatase alcaline thermostable (Challier, 1978) dans le placenta humain perfusé. La concentration fcetale représente généralement quelques pour cent de la concentration maternelle. Elle n'atteint jamais les 30 à $50 \%$ qu'on devrait obtenir si la partition des sécrétions était basée uniquement sur le rapport des débits circulatoires. Certains stéroïdes font exception à cette règle notamment l'œstrone (Gurpide et al., 1982) et la cortisone (Dancis et al., 1978) que le placenta produit à partir de précurseurs maternels et qu'il libère dans la circulation fœtale. La 
libération préférentielle des sécrétions placentaires vers la circulation maternelle peut être expliquée par la proximité du trophoblaste et de la circulation maternelle, et par l'importance du débit circulatoire utéroplacentaire. L'existence d'une barrière fœtale localisée entre le trophoblaste et le sang fœtal peut également être avancée. On remarquera cependant que si une telle barrière existe, elle n'affecte apparemment pas les produits de transformation de certains stéroïdes maternels qui apparaissent dans la circulation fœtale.

\section{Catabolisme}

\section{Nutriments}

Le placenta détourne une partie importante des nutriments qu'il capte dans la circulation utérine pour son propre métabolisme. Dans l'espèce humaine, les quantités de glucose et d'oxygène ainsi prélevées sont estimées à 60 et à $20 \%$ (Hauguel et al., 1986; Challier et al., 1976). Une proportion de glucose voisine est catabolisée par le placenta chez la brebis (Hay et al., 1983). Cette préemption constitue une véritable barrière métabolique pour l'acide glutamique dont la captation maternelle est importante, la vitesse d'efflux fœtale faible, et le taux intraplacentaire élevé, et qui subit en outre une transformation locale en glutamine (Schneider et al., 1979).

\section{Hormones et substances exogènes}

La capacité d'oxydation du placenta, quoique faible comparée au foie adulte n'est pas négligeable. Les médicaments peuvent subir des réactions d'inactivation telles que la glucuronidation, la sulfation, la mono-oxygénation dépendante du cytochrome P450, etc. (Juchau \& FaustmanWatts, 1983). Dans le cas des benzopyrènes, des hydrocarbures polycycliques issus des cigarettes, une hydroxylase induite dans le placenta forme des métabolites intermédiaires, notamment des époxydes, qui à la différence de leur précurseur ont des potentialités carcinogènes (Pelkonen, 1984). L'activité barrière peut alors se doubler d'une activité toxique.

Une barrière catabolique a été démontrée pour un certain nombre d'hormones : les catécholamines, par l'intermédiaire des activités MAO et COMT (Nandakumaran et al., 1983), les glucocorticoïdes (Dancis et al., 1978) et l'insuline (Posner, 1973).

\section{SÉGRÉGATION DES RÉCEPTEURS ET DES TRANSPORTEURS}

\section{Transporteurs}

Les transporteurs les mieux connus sont ceux du glucose et des acides aminés. Les transporteurs du glucose sont détectés à la fois sur la face fcetale et sur la face maternelle du trophoblaste. La captation du glucose radioactif depuis la circulation foetale ou la circulation maternelle du cobaye est identique (Yudilevitch et al., 1979). De plus, les microvésicules préparées à partir des microvillosités (Johnson \& Smith, 1980) ou de la membrane plasmique basale (Johnson \& Smith, 1985) du syncytium ont des caractéristiques cinétiques similaires. II n'y a ni ségrégation des transporteurs, ni asymétrie fonctionnelle. Le sens du gradient de concentration conditionne le sens du transport.

Les acides aminés comme la phénylalanine ont une captation similaire dans le 
Côté foetal

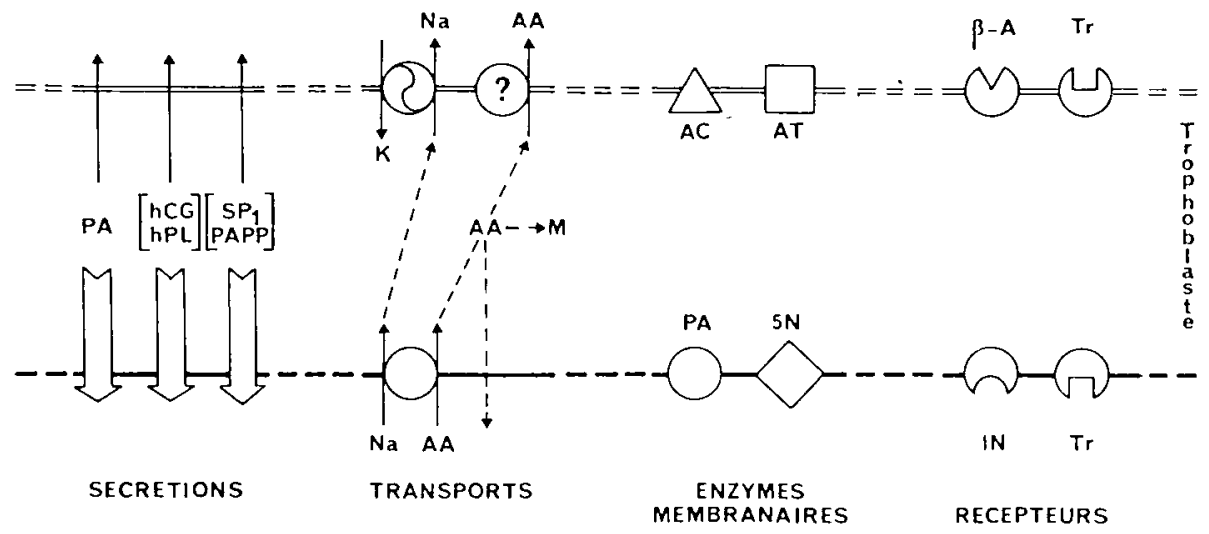

Còté Maternel

Fig. 2. Polarisation fonctionnelle du syncytium trophoblastique humain. $A A$ : acide aminé; $A C$ : adényl cyclase; AT : adénosine triphosphatase; B-A : récepteur B-adrénergique; hCG : gonadotropine chorionique humaine; $\mathrm{hPL}$ : hormone placentaire lactogène; In : récepteur insulinique; $\mathrm{K}$ : potassium; $M$ : voies métaboliques; $5 \mathrm{~N}$ : 5 -nucléotidase; $\mathrm{Na}$ : sodium; $\mathrm{PA}$ : phosphatase alcaline thermostable; PAPP : protéine plasmatique de gestation $\mathrm{A} ; \mathrm{SP} 1$ : B1-glycoprotéine de gestation; $\mathrm{Tr}$ : récepteur à la transferrine.

placenta de cobaye qu'ils soient injectés dans la circulation fœtale ou maternelle (Fig. 2). Mais l'efflux dans la circulation foetale est plus important que dans la circulation maternelle (Eaton \& Yudilevitch, 1981). Une telle dissymétrie entre les vitesses d'efflux fœtale et maternelle constitue la base du transport actif secondaire (conséquence du transport actif de $\mathrm{Na}$ ) de certains acides aminés.

\section{Enzymes et récepteurs}

La composition en protéine de la membrane des microvillosités syncytiales diffère de celles de la membrane plasmique basale (Kelley et al., 1983). Elle se traduit par une répartition asymétrique des récep- teurs et des enzymes sur les 2 faces du syncytium.

Sur la membrane apicale du trophoblaste (face maternelle) se trouvent de très nombreux récepteurs; récepteurs à l'insuline, à la transferrine, à la transcobalamine, aux facteurs de croissance épidermique (EGF) et insuliniques (IGF), aux lipoprotéines de basse densité (LDL) et aux immunoglobulines $\mathrm{G}$ ( $\mathrm{lgG}$ ) (Truman \& Ford, 1984). Les enzymes sont la phosphatase alcaline et la [5-nucléotidase]. Sur la membrane plasmique basale du trophoblaste (face fœtale) sont présents des récepteurs aux substances $\beta$-adrénergiques (Whitsett et al., 1980) et à la transferrine (Vanderpuye et al., 1986). Les enzymes sont l'adénosine triphosphatase et l'adényl-cyclase (Whitsett et al., 1979; Kelley et al., 1983). 


\section{CONCLUSION}

La membrane placentaire se présente comme une série de barrières trophoblastique et endothéliale plus ou moins efficaces. Ces barrières sont percées de pores de taille différente selon les assises. Certaines de ces barrières, en particulier le glycocalyx et les lames basales, ont un effet électrostatique. Le métabolisme local, les histiocytes, certaines vacuoles lipidiques participent à l'effet de barrière. La filtration des molécules selon la taille des pores et la répulsion électrostatique des charges anioniques sont parmi les effets les mieux connus. Une barrière métabolique spécifique affecte les nutriments, certaines hormones et médicaments.

La barrière placentaire du placenta épithéliochorial apparaît beaucoup plus impénétrable que celle des placentas hémochoriaux, confirmant ainsi les observations de Flexner et al. (1942). La barrière hémochoriale est au contraire éminemment permissive, puisqu'elle laisse passer des substances de la taille de l'albumine.

L'asymétrie dans la distribution des enzymes des transporteurs et des récepteurs est commune à la plupart des épithéliums. Elle a des conséquences fonctionnelles, qui apparaissent en partie dans le transport des acides aminés. Mais les interactions entre les systèmes de communication, le métabolisme et le transport résultant de la polarisation du trophoblaste sont encore méconnus.

\section{RÉFÉRENCES}

Aoki A., Metz J., Forssmann W.G. (1978) Studies on the ultrastructure and permeability of the hemotrichorial placenta. Cell Tissue Res. 192, 409-422
Bara M., Challier J.C. \& Guiet-Bara A. (1988) Membrane potential and input resistance in syncytiotrophoblast of human term placenta in vitro. Placenta 9, 139-146

Battaglia F.C., Behrman R.E., Meschia G., Seeds A.E. \& Bruns P.D. (1968) Clearance of inert molecules, $\mathrm{Na}$, and $\mathrm{Cl}$ - ions across the primate placenta. Am. J. Obstet. Gynecol. 102, 1135-1143

Behre A., Bardsley W.G., Harkes A. \& Sibley C.P. (1987) Molecular charge effects on the protein permeability of the guinea-pig placenta. Placenta 8, 365-380

Bersinger N.A., Schneider H. \& Keller P.J. (1986) Synthesis of placental proteins by the human placenta perfused in vitro. Gynecol. Obstet. Invest. 22, 47-51

Bissonnette J., Cronan J.Z., Richards L.L. \& Wickham W.K. (1979) Placental transfer of water and non-electrolytes during a single circulatory passage. Am. J. Physiol. 236, C47-C52

Boyd R.D.H., Haworth C., Stacey T.E. \& Ward R.H.T. (1976) Permeability of the sheep placenta to unmetabolizable polar non-electrolytes. $J$. Physiol. (London) 256, 617-634

Challier J.C. (1978) Analyse expérimentale des échanges materno-fœtaux dans le placenta humain perfusé in vitro. Thèse de doctorat ès sciences université Pierre-et-Marie-Curie, Paris

Challier J.C., Schneider H. \& Dancis J. (1976) in vitro perfusion of human placenta. V. Oxygen consumption. Am. J. Obstet. Gynecol. 126, 261265

Challier J.C., Guerre-Millo M., Nandakumaran M., Gerbaut L. \& D'Athis P. (1985) Clearance of compounds of different molecular size in the human placenta in vitro. Biol. Neonate 48, 143-148

Conrad E.E. \& Faber J.J. (1977) Water and electrolyte acquisition across the placenta of the sheep. Am. J. Physiol. 233, H475-H487

Dancis J., Jansen V., Kayden H., Bjornson L. \& Levitz M. (1974) Transfer across perfused human placenta. III. Effect of chain lenght on transfer of free fatty acids. Pediatr. Res. 8, 796-799

Dancis J., Jansen V., Levitz M. \& Rosner W. (1978) Effect of protein binding on transfer and metabolism of cortisol in perfused human placenta. J. Clin. Endocrinol. Metab. 46, 863-868

Dancis J., Kammerman S., Jansen V., Schneider H. \& Levitz M. (1981) Transfer of urea, so- 
dium and chloride across the human perfused placenta. Am. J. Obstet. Gynecol. 141, 677-681

De Virgilis G., Sideri M., Fumagalli G. \& Remotti G. (1982) The junctional pattern of the human villous trophoblast. A freeze-fracture study. Gynecol. Obstet. Invest. 14, 263-272

Eaton B.M. \& Yudilevitch D. (1981) Uptake and asymmetric efflux of amino acids at the maternal and fetal sides of placenta. Am. J. Physiol. 241, C106-C112

Faber J.J. (1973) Diffusional exchange between fœtus and mother as a function of the physical properties of the diffusing materials. In : Fotal and Neonatal Physiology. (Comline K.S., Cross K.W., Oawes G.S. \& Nathanielsz P.W., eds) Cambridge University Press, London, pp. 306327

Faber J.J. \& Hart F.M. (1966) The rabbit placenta as an organ of diffusional exchange : comparison with other species by dimensional analysis. Circ. Res. 19, 816-833

Faber J.J., Green T.J. \& Long L.R. (1971) Permeability of the rabbit placenta to large molecules. Am. J. Physiol. 220, 688-693

Farquhar M.G., Courtoy P.J., Lemkin M.C. \& Kanwar Y.S. (1982) Current knowledge of the functional architecture of the glomerular basement membrane. In : New Trends in Basement Membrane Research. (Kuehn K., Schoene H. \& Timp L., eds) Raven Press, New York, pp. 9-29

Firth J.A., Bauman K.F. \& Sibley C.P. (1983) The intercellular junctions of guinea-pig placental capillaries : a possible structural basis for endothelial solute permeability. J. Ultrastruct. Res. 85, 45-57

Flexner L.B. \& Gellhorn A. (1942) The comparative physiology of placental transfer. Am. J. Obstet. Gynecol. 43, 965

Fox H. (1967) The incidence and significance of vasculo-syncytial membranes in the human placenta. J. Obstet. Gynecol. Br. Commonw. 74, 28-33

Fox H. (1978) Pathology of the Placenta. W.B. Saunders, London, pp. 20

Gammal E.B. (1985) Syncytial channels in the villous trophoblast of the macaque. J. Anat. 141, 181-191

Grosser O. (1909) Vergleichende Anatomie und Entwicklungsgeschichte der Eihäute und der Placenta. W. Braumüller, Vienna \& Leipzig, pp. 291-292
Grosser O. (1927) Frühentwicklung, Eihautbildung und Placentation des Menschen und der Säugetiere. Bergmann J.F., Munich

Guerre-Millo M., Challier J.C., Rey E., Nandakumaran M., Richard M.O. \& Olive G. (1982) Materno-fetal transfer of two benzodiazepines. Effect of protein binding and placental uptake. Dev. Pharmacol. Ther. 4, 158-172

Gurpide E., Marks C., De Zieglẹ D., Berk P.D. \& Brandes J.M. (1982) Asymmetric release of estrone and estradiol derived from labeled precursors in the perfused human placentas. Am. J. Obstet. Gynecol. 144, 551-555

Hauguel S., Desmaizieres V. \& Challier J.C. (1986) Glucose uptake, utilization, and transfer by the human placenta as functions of maternal glucose concentration. Pediatr. Res. 20, 269273

Hay W.W., Sparks J.W., Wilkening R.B., Battaglia F.C. \& Meschia G. (1983) Partition of maternal glucose production between conceptus and maternal tissues in sheep. Am. J. Physiol. 245, E347-E350

Hedley R. \& Bradbury M.W.B. (1980) Transport of polar non-electrolytes across the intact and perfused guinea-pig placenta. Placenta 1, 277285

Heinrich D., Metz J., Raviola E. \& Forssmann W.G. (1976) Ultrastructure of perfusion-fixed fetal capillaries in the human placenta. Cell Tissue Res. 172, 157-169

Johnson L.W. \& Smith C.H. (1980) Monosaccharide transport across microvillous membrane of human placenta. Am. J. Physiol. 238, C160C168

Johnson L.W. \& Smith C.H. (1985) Glucose transport across the basal plasma membrane of human placental syncytiotrophoblast. Biochim. Biophys. Acta 815, 44-50

Juchau M.R. \& Faustman-Watts E. (1983) Pharmacokinetic considerations in the maternalplacental-fetal unit. Clin. Obstet. Gynecol. 26, 379-390

Kaufmann P. \& Davidoff M. (1977) The guineapig placenta. Adv. Anat. Embryol. Cell Biol. 53, 1-91

Kaufmann P., Schroder H. \& Leichtweiss H.P. (1982) Fluid shift across the placenta. II. Fetomaternal transfer of horseradish peroxidase in the guinea pig. Placenta 3, 339-348 
Kaufmann P., Schroder H., Leichtweiss H.P. \& Weinterhager E. (1987) Are there membranelined channels through the trophoblast ? A study with lanthanum hydroxide. Trophoblast Res. 2, 557-571

Kawagoe K., Kawana T. \& Sakamoto S. (1981) Negative surface charge of abnormal human trophoblast. Acta Histochem. Cytochem. 14, 554-560

Kelley L.K., Smith C.H. \& King B. (1983) Isolation and partial characterization of the basal cell membrane of the human placenta. Biochim. Biophys. Acta 734, 91-98

King B.P. (1981) The distribution and mobility of anionic sites on the surface of human placental syncytial membrane. Anat. Rec. 199, 15-22

King B.F. (1985) Distribution and characterization of anionic sites in the trophoblast and capillary basal laminas of human placental villi. Anat. Rec. 212, 63-68

Martin B.J., Spicer S.S. \& Smythe N.M. (1974) Cytochemical studies on the maternal surface of syncytiotrophoblast of human early and term placenta. Anat. Rec. 178, 769-786

Metz J. (1980) On the developping rat placenta. I. Differentiation and junctional alterations of labyrinthine layers II and III. Anat. Embryol. 159, 289-305

Metz J., Weihe E., Heinrich D. (1979) Intercellular junctions in the full term human placenta. Anat. Embryol. 158, 41-50

Meschia G, Battaglia F.C. \& Bruns P.D. (1967) Theoritical and experimental study of transplacental diffusion. J. Appl. Physiol. 22, 1171-1178

Moll W. \& Kastendieck E. (1977) Transfer of $\mathrm{N}_{2} \mathrm{O}, \mathrm{CO}, \mathrm{HTO}$ in the artificially perfused guinea-pig placenta. Resp. Physiol. 29, 283-302

Nandakumaran M., Gardey C., Challier J.C. \& Olive G. (1983) Placental monoamine oxidase content and inhibition : effect of enzyme inhibition on materno-fetal transfer of noradrenaline (norepinephrine) in the human placenta in vitro. Placenta 4, 57-64

Nelson D.M., Smith C.H., Enders A.C. \& Donohue T.M. (1976) The non-uniform distribution of acidic components on the human placental syncytial trophoblast surface membrane : a cytochemical and analytical study. Anat. Rec. 184, 159-182

Pelkonen O. (1984) Detoxification and toxification process in the human feto-placental unit.
Drugs and pregnancy. In : Maternal Drug Handling-Fetal Drug Exposure. (Krauer B., Krauer F., Hytten F.E. \& Del Pozo E., eds) Academic Press, London, pp. 63-72

Posner B.I. (1973) Insulin metabolizing enzyme activities in human placental tissue. Diabetes 22, 552-560

Reale E., Wang T., Zaccheo D., Maganza C. \& Pescetto G. (1980) Junctions on the maternal blood surface of the human placental syncytium. Placenta 1, 245-258

Schennan D.B. \& Boyd C.A.R. (1987) lon transport by the placenta : a review of membrane transport systems. Biochim. Biophys. Acta 906, 437-457

Schneider H., Mohlen K.H., Challier J.C. \& Dancis J. (1979) Transfer of glutamic acid across the human placenta perfused in vitro. Br. J. Obstet. Gynecol. 86, 299-306

Sibley C.P., Bauman K.F. \& Firth J.A. (1981) Ultrastructural study of the permeability of the guinea-pig placenta to horseradish peroxidase. Cell Tissue Res. 219, 637-647

Sibiey C.P., Bauman K.F. \& Firth J.A. (1983) Molecular charge as a determinant of macromolecule permeability across the fetal capillary endothélium of the guinea-pig placenta. Cell Tissue Res. 229, 365-377

Sideri M., De Virgilis G., Rainoldi R. \& Remotti G. (1982) Peculiar ruthenium red staining pattern of the human early trophoblast. Experientia $38,847-848$

Sideri M., De Virgilis G., Rainoldi R. \& Remotti G. (1983) The ultrastructural basis of the nutritional transfer : evidence of different patterns in the plasma membranes of the multilayered placental barrier. Trophoblast. Res. 1, 15-26

Sideri M., Zannoni E. \& Challier J.C. (1987) Transfer of horseradish peroxidase across the human placental cotyledon perfused in vitro. Trophoblast Res. 2, 573-584

Snoeck J. (1958) Le Placenta Humain. Masson Ed., Paris

Solomon A.K. (1968) Characterization of biological membranes by equivalent pores. J. Gen. Physiol. 51, 335-364

Stenseth K., Hedin U. \& Thyberg J. (1983) Endocytosis, intracellular transport, and turnover of anionic and cationic proteins in cultured mouse peritoneal macrophages. Eur. J. Cell Biol. 31, 15-25 
Stulc J. (1985) Validity of the equivalent pore model in placental physiology. Contrib. Obstet. Gynecol. 13, 85-91

Stulc J., Friederich R. \& Jiricka Z. (1969) Estimation of the equivalent pore dimensions of the rabbit placenta. Life Sci. 8, 167-180

Taylor A.E. \& Granger D.N. (1983) Equivalent pore modeling : vesicles and channels. Fed. Proc. 42, 2440-2445

Thornburg K.L. \& Faber J.J. (1976) The steady state concentration gradients of an electrondense marker (ferritin) in the three-layered hemochorial placenta of the rabbit. J. Clin. Invest. 58, 912-925

Thornburg K.L., Binder N. \& Faber J.J. (1979) Distribution of ionic sulfate, lithium, and bromide across the sheep placenta. Am. J. Physiol. 5, C58-C65

Truman P. \& Ford H.C. (1984) The brush border of the human term placenta. Biochim. Biophys. Acta 779, 139-160

Vanderpuye O.A., Kelley L.K. \& Smith C.H. (1986) Transferrin receptors in the basal plasma membrane of the human placental syncytiotrophoblast. Placenta 7, 391-403

Wada H.G., Gornicki S.Z. \& Susmann H.H. (1977) The sialoglycoprotein subunits of human placental brush border membranes characterized by two dimensional eletrophoresis. J. Supramol. Struct. 6, 473-474
Whyte A. (1980) Lectin binding by microvillous membranes and coated-pit regions of human syncytial trophoblast. Histochem. J. 12, 599-607

Whyte A. \& Locke Y.W. (1978) Increased sialylation of surface glycopeptides of human trophoblast compared with fetal cells from the same conceptus. J. Exp. Med. 148, 1087-1092

Whitsett J.A., Johnson C.L. \& Hawkins K. (1979) Difference in localization of insulin receptors and adenylate cyclase in the human placenta. Am. J. Obstet. Gynecol. 133, 204-207

Whitsett J.A., Johnson C.L., Noguchi A., Darovec-Beckerman C. \& Costello M. (1980) Badrenergic receptors and catecholamine sensitive adenylate cyclase of the human placenta. $J$. Clin. Endocrinol. Metab. 50, 27-32

Wilkening R.B., Anderson S., Marteson L. \& Meschia G. (1982) Placental transfer as function of uterine blood flow. Am. J. Physiol. 242, 429-436

Winkel C.A., Snyder J.M., MacDonald P.C. \& Simpson E.R. (1980) Regulation of cholesterol and progesterone synthesis in human placental cells in culture by serum lipoproteines. Endocrinology 106, 1054-1060

Yudilevitch D.L., Eaton B.M., Short A.H. \& Leichtweiss H.P. (1979) Glucose carriers at maternal and fetal sides of the trophoblast in the guinea-pig placenta. Am. J. Physiol. 237, C205$\mathrm{C} 212$ 